\title{
Study on Motion Mathematical Model of Four Rotor UAV
}

\author{
Ling Yehua
}

Guangxi Normal University for Nationalities, Guangxi, Chongzuo, China

Keywords: Four Rotor, Uav, Mathematical Model

\begin{abstract}
Four rotor UAV has the characteristics of small volume, simple construction, light weight, low cost and good invisibility. It can be adapted to multi-platform and multi-dimensional environment. It has the lower flight height and the very good mobility. It can have the flexible rising and landing on a relatively small platform. It doesn't need the ejection device, launcher and other auxiliary devices. Based on its many advantages, it has very broad application prospects both in military and civil fields. We mainly take the four rotor UAV as the research object to establish the kinematic and dynamic mathematical model of four rotor UAV and give the definition of usual coordinates for four rotor UAV, and then give the conversion relations of all vectors for four rotor UAV in inertial frame and body frame in the paper. We adopt Newton - euler method to obtain the rigid body dynamic equations of four rotor UAV model in the paper. We further analyze all kinds of forces and moments which act on the four rotor UAV in details again in the paper. We deduce the complete expression of overall mathematical model for four rotor UAV and make it be simplified in the paper.
\end{abstract}

\section{Introduction}

A four rotor is composed of four DC motors and propellers fixed on the motor, and the simple schematic diagram is shown in Figure 1. By the rotation of four rotor wings four rotor UAV can produce the control action. Among them, motor $M_{1}$ and motor $M_{3}$ have the clockwise rotations respectively with the angular velocity $\omega_{1}$ and the angular velocity $\omega_{3}$ to respectively generated the thrust $f_{1}$ and the thrust $f_{3}$. At the same time, the other two motors $\left(M_{2}\right.$ and $M_{4}$ ) have the counter-clockwise rotations respectively with the angular velocity $\omega_{2}$ and the angular velocity $\omega_{4}$ to respectively generated the thrust $f_{2}$ and the thrust $f_{4}$.

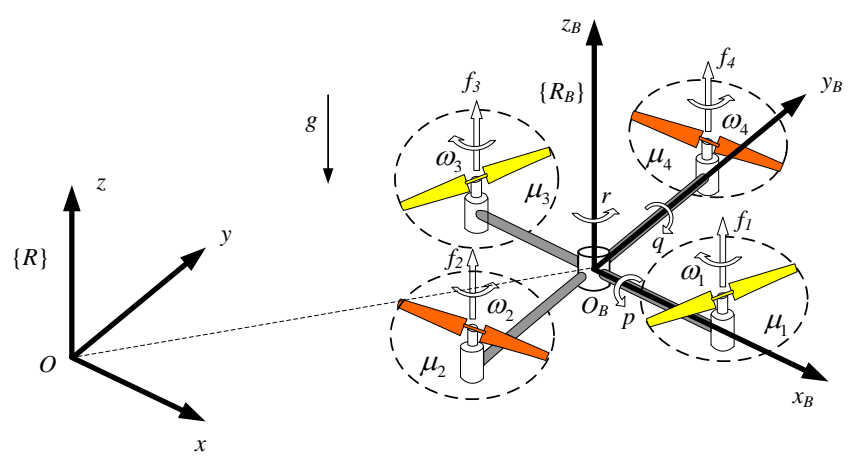

Figure 1The Schematic Diagram of Four Rotor UAV

In order to to control the four rotor UAV, we need to establish the mathematical model of UAV in the paper. We make use of Newton - Euler method to establish the model of four rotor UAV so as to take it as the basis of fault diagnosis and fault-tolerant control study in the paper. 


\section{Kinematical Equations}

In order to control the aerial vehicle, we need to define the spatial reference coordinate system, otherwise it is impossible to describe the attitude and position of aerial vehicle. At the same time we need to define the body frame which is attached to the aerial vehicle. The relative relation between two coordinate systems describes the motion state of aerial vehicle. In Figure 1, Oxyz is inertial frame. $O_{B} X_{B} y_{B} z_{B}$ is body frame. The origin $O_{B}$ of body frame is attached to a certain point of UAV (Usually we take its centroid as the certain point).

The movement of UAV includes translational motion and rotation itself. We use its centroid position and speed to represent the translational motion of UAV. We use $p_{o}^{I}=\left[\begin{array}{lll}x & y & z\end{array}\right]^{T}$ to show the position of body frame origin $O_{B}$ in the inertial frame. We use $v_{o}^{I}=\left[\begin{array}{lll}\dot{x} & \dot{y} & \dot{z}\end{array}\right]^{T}$ to show the velocity vector of UAV in the inertial frame. We use $v_{o}^{B}=\left[\begin{array}{lll}u & v & w\end{array}\right]^{T}$ to show the velocity vector of $\mathrm{UAV}$ in the body frame. In order to represent the rotation of UAV, we adopt three eulerian angles, $\theta \theta, \psi \psi$ to show the direction of relative inertial frame in the body frame. We use the velocity vector $\omega^{B}=\left[\begin{array}{lll}p & q & r\end{array}\right]^{T}$ to show the instantaneous angular velocity of each axis in the body frame. Among them, the roll angle $\phi$ is the included angle between UAV symmetry plane and plumb line plane with the axes. When it rolls over to the right, the formed angle is positive. The angle of pitch $\theta$ is the included angle between body axis $O x_{B}$ and horizontal plane $O x y$. When the head of UAV is upward, the formed angle is positive. The yaw angle $\psi$ is the included angle between the projection of body axis $O x_{B}$ and horizontal plane $O x y$ and $O x_{B}$ axis. When UAV yaws to the right, the formed angle is positive.

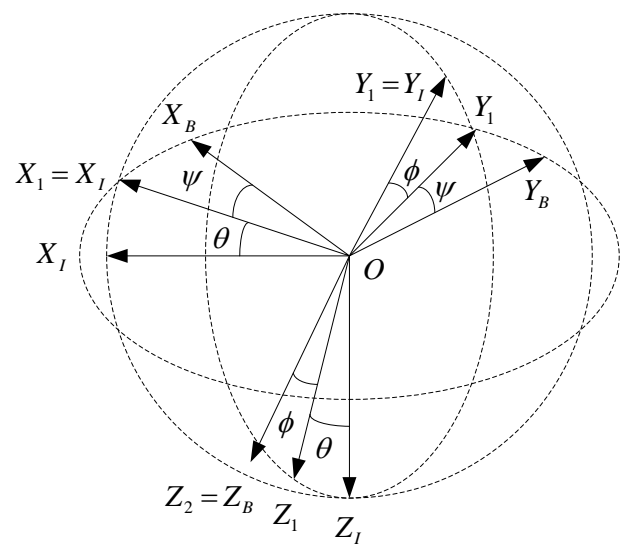

Figure 2 The Conversion of Coordinate System

We have known the vector $v_{o}^{B}$ of the body frame, then we can represent the vector $v_{o}^{I}$ in the inertial frame as

$$
v_{o}^{I}=R v_{o}^{B}
$$

In order to find the transformation matrix $R$, we adopt the right hand rule ( according to the order of $z \rightarrow y \rightarrow x$ axis rotation ). It is shown in Figure 2. The corresponding matrixes produced by the rotation is shown as follows each time.

$$
\begin{aligned}
R_{z}(\psi) & =\left[\begin{array}{ccc}
C_{\psi} & -S_{\psi} & 0 \\
S_{\psi} & C_{\psi} & 0 \\
0 & 0 & 1
\end{array}\right] \\
R_{y}(\theta) & =\left[\begin{array}{ccc}
C_{\theta} & 0 & S_{\theta} \\
0 & 1 & 0 \\
-S_{\theta} & 0 & C_{\theta}
\end{array}\right]
\end{aligned}
$$




$$
R_{x}(\phi)=\left[\begin{array}{ccc}
1 & 0 & 0 \\
0 & C_{\phi} & -S_{\phi} \\
0 & S_{\phi} & C_{\phi}
\end{array}\right]
$$

Among them, $S_{(\cdot)}, C_{(\cdot)}$ and $T_{(\cdot)}$ represent $\sin (\cdot), \cos (\cdot)$ and $\tan (\cdot)$ respectively.

Therefore, the expression of transformation matrix can be written as

$$
\begin{aligned}
R & =R_{x}(\phi) R_{y}(\theta) R_{z}(\psi) \\
R & =\left[\begin{array}{ccc}
C_{\theta} C_{\psi} & C_{\psi} S_{\theta} S_{\phi}-C_{\phi} S_{\psi} & C_{\phi} C_{\psi} S_{\theta}+S_{\phi} S_{\psi} \\
C_{\theta} S_{\psi} & S_{\theta} S_{\phi} S_{\psi}+C_{\phi} C_{\psi} & C_{\phi} S_{\theta} S_{\psi}-C_{\psi} S_{\phi} \\
-S_{\theta} & C_{\theta} S_{\phi} & C_{\theta} C_{\phi}
\end{array}\right]
\end{aligned}
$$

Among them, $R$ is the transformation matrix from body frame to inertial frame. Among them, $R$ is the orthogonal matrix, and it satisfies the relation $R^{-1}=R^{T}$.

Therefore, the kinematics equations of position can be written in component form as follows.

$$
\begin{aligned}
& \dot{x}=u C_{\theta} C_{\psi}+v\left(C_{\psi} S_{\theta} S_{\phi}-C_{\phi} S_{\psi}\right)+w\left(C_{\phi} C_{\psi} S_{\theta}+S_{\phi} S_{\psi}\right) \\
& \dot{y}=u C_{\theta} S_{\psi}+v\left(S_{\theta} S_{\phi} S_{\psi}+C_{\phi} C_{\psi}\right)+w\left(C_{\phi} S_{\theta} S_{\psi}-C_{\psi} S_{\phi}\right) \\
& \dot{z}=-u S_{\theta}+v C_{\theta} S_{\phi}+w C_{\theta} C_{\phi}
\end{aligned}
$$

Similarly, according to the definition, we can know that the transformational relation of angular velocity is shown as follows.

$\dot{\Theta}=T \omega^{B}$

In order to find the transformation matrix of euler angle, we do the following rotation changeto obtain the following equations.

$$
\begin{aligned}
& \omega^{B}=\left[\begin{array}{c}
\dot{\phi} \\
0 \\
0
\end{array}\right]+R_{x}^{T}(\phi)\left[\begin{array}{l}
0 \\
\dot{\theta} \\
0
\end{array}\right]+R_{x}^{T}(\phi) R_{y}^{T}(\theta)\left[\begin{array}{c}
0 \\
0 \\
\dot{\psi}
\end{array}\right]=T^{-1} \Theta \\
& T^{-1}=\left[\begin{array}{ccc}
1 & 0 & -S_{\theta} \\
0 & C_{\phi} & S_{\phi} C_{\theta} \\
0 & -S_{\phi} & C_{\phi} C_{\theta}
\end{array}\right] \\
& T=\left[\begin{array}{ccc}
1 & S_{\phi} T_{\theta} & C_{\phi} T_{\theta} \\
0 & C_{\phi} & -S_{\phi} \\
0 & S_{\phi} & C_{\phi} / C_{\theta}
\end{array}\right]
\end{aligned}
$$

Therefore, the kinematical equation of euler angle is shown as follows.

$$
\begin{aligned}
& \dot{\phi}=p+q S_{\phi} T_{\theta}+r C_{\phi} T_{\theta} \\
& \dot{\theta}=q C_{\phi}-r S_{\phi} \\
& \dot{\psi}=\frac{1}{C_{\theta}}\left[q S_{\phi}+r C_{\phi}\right]
\end{aligned}
$$

\section{Dynamical Equations}

We have two types of methods to derive the rigid body dynamics mainly, namely newton - euler 
method and the euler - lagrange method. In newton - euler method, dynamics is established under the body frame, and the coordinate system transformation used afterwards makes it be expressed in the inertial frame. On the contrary, the euler - lagrange method needs us to use the generalized coordinates (inertial frame) directly. Because the newton - euler method is simple and intuitive, we adopt the newton - euler method to derive the rigid body dynamics of four rotor model.

We use $m$ to show the quality of the whole rotor aerial vehicle, and we define the inertial matrix in body frame as follows.

$$
I=\left[\begin{array}{ccc}
I_{x x} & -I_{x y} & -I_{x z} \\
-I_{y x} & I_{y y} & -I_{y z} \\
-I_{z x} & -I_{z y} & I_{z z}
\end{array}\right]
$$

Among them, , $I_{y y} I_{y y}, I_{z z} I_{z z}$ are the moments of inertia of each principal axis, and , $I_{x z}=I_{z x} I_{x z}=I_{z x}, I_{y z}=I_{z y} I_{y z}=I_{z y} \quad$ are the products of inertia.

We set $r_{g}^{B}=\left[\begin{array}{lll}x_{g} & y_{g} & z_{g}\end{array}\right]^{T}$ as the deviation between the origin of body frame and the four rotor centroid in body frame.

According to newton's second law, we can obtain

$$
m \cdot \dot{v}_{c}^{I}=F_{c}^{I}=R F_{c}^{B}
$$

Among them, $v_{c}^{I}=\left[\begin{array}{lll}\dot{x} & \dot{y} & \dot{z}\end{array}\right]^{T}$ is the existence deviation $r_{g}^{B}$, the projection of linear velocity of the origin in inertial frame, and $F_{c}^{I}, F_{c}^{B}$ are the acting forces under the inertial frame and body frame respectively.

According to the velocity composition theorem, when there is the deviation $r_{g}^{B}$, the linear velocity in body frame is

$$
v_{c}^{B}=v_{o}^{B}+\omega^{B} \times r_{g}^{B}
$$

When it is projected onto the inertial frame:

$$
v_{c}^{I}=R v_{c}^{B}=R\left(v_{o}^{B}+\omega^{B} \times r_{g}^{B}\right)
$$

When we take a derivative with respect to the equation above, we can get

$$
\begin{aligned}
\dot{v}_{c}^{I} & =R\left(\dot{v}_{o}^{B}+\dot{\omega}^{B} \times r_{g}^{B}+\omega^{B} \times \dot{r}_{g}^{B}\right)+\dot{R}\left(v_{o}^{B}+\omega^{B} \times r_{g}^{B}\right) \\
& =R\left(\dot{v}_{o}^{B}+\dot{\omega}^{B} \times r_{g}^{B}\right)+R S\left(\omega^{B}\right)\left(v_{o}^{B}+\omega^{B} \times r_{g}^{B}\right) \\
& =R\left(\dot{v}_{o}^{B}+S\left(\dot{\omega}^{B}\right) r_{g}^{B}+S\left(\omega^{B}\right) v_{o}^{B}+S^{2}\left(\omega^{B}\right) r_{g}^{B}\right)
\end{aligned}
$$

Among them, the anti-symmetric matrix

$$
\begin{aligned}
& S(\xi)=-S^{T}(\xi)=\left[\begin{array}{ccc}
0 & -\xi_{3} & \xi_{2} \\
\xi_{3} & 0 & -\xi_{1} \\
-\xi_{2} & \xi_{1} & 0
\end{array}\right] \\
& \xi=\left[\begin{array}{lll}
\xi_{1} & \xi_{2} & \xi_{3}
\end{array}\right]^{T}
\end{aligned}
$$

The operation of anti-symmetric matrix:

$$
\xi \times a=S(\xi) a
$$

And the ordinary differential equation of rotation matrix is

$\dot{R}=R S\left(\omega^{B}\right)$

We substitute the equation (17) into the equation (14), so we obtain

$m\left(\dot{v}_{o}^{B}+S\left(\dot{\omega}^{B}\right) r_{g}^{B}+S\left(\omega^{B}\right) v_{o}^{B}+S^{2}\left(\omega^{B}\right) r_{g}^{B}\right)=F_{c}^{B}=F_{o}^{B}$

According to the moment of momentum theorem, we can know

$\tau_{o}^{B}=\dot{H}_{o}^{B}=\dot{H}_{c}^{B}+\omega^{B} \times H_{c}^{B}$ 
Among them, $H_{o}^{B}$ is the angular momentum under the body frame, $H_{c}^{B}=I \cdot \omega^{B}$ is the angular momentum which takes the file:/C: $\backslash$ Program Files\Youdao\Dict4\resultui\queryresult.htmlcentroid as the origin, and $\tau_{o}^{B}=\left[\begin{array}{lll}K & M & N\end{array}\right]^{T}$ is the applied moment in the body frame.

According to the equation (16), when we obtain the existence deviation $r_{g}^{B}$, the accelerated velocity of origin in the body frame is

$a_{c}^{B}=\dot{v}_{o}^{B}+\omega^{B} \times v_{o}^{B}$

The additional moment produced when the origin is in the body frame is

$$
\begin{aligned}
\tau_{c}^{B} & =r_{g}^{B} \times m a_{c}^{B}=r_{g}^{B} \times m\left(\dot{v}_{o}^{B}+\omega^{B} \times v_{o}^{B}\right) \\
& =m \cdot r_{g}^{B} \times \dot{v}_{o}^{B}+m \cdot r_{g}^{B} \times\left(\omega^{B} \times v_{o}^{B}\right)
\end{aligned}
$$

According to the equation (23) and the equation (25), we can obtain :

$\dot{H}_{c}^{B}+\omega^{B} \times H_{c}^{B}+r_{g}^{B} \times m a_{c}^{B}=\tau_{o}^{B}$

When we arrange them, we can obtain:

$I \cdot \omega^{B}+\omega^{B} \times I \cdot \omega^{B}+m \cdot r_{g}^{B} \times \dot{v}_{o}^{B}+m \cdot r_{g}^{B} \times\left(\omega^{B} \times v_{o}^{B}\right)=\tau_{o}^{B}$

Then we can obtain the following equation according to the definition of anti-symmetric matrix.

$I \dot{\omega}^{B}+S\left(\omega^{B}\right) I \omega^{B}+m S\left(r_{g}^{B}\right) \dot{v}_{o}^{B}+m S\left(r_{g}^{B}\right) S\left(\omega^{B}\right) v_{o}^{B}=\tau_{o}^{B}$

The component forms of the two equations above are shown as follows.

$$
\begin{gathered}
m\left[\dot{u}-v r+w q-x_{g}\left(q^{2}+r^{2}\right)+y_{g}(p q-\dot{r})+z_{g}(p r+\dot{q})\right]=F_{x} \\
m\left[\dot{v}-w p+u r-y_{g}\left(r^{2}+p^{2}\right)+z_{g}(q r-\dot{p})+x_{g}(q p+\dot{r})\right]=F_{y} \\
m\left[\dot{w}-u q+v p-z_{g}\left(p^{2}+q^{2}\right)+x_{g}(r p-\dot{q})+y_{g}(r q+\dot{p})\right]=F_{z} \\
I_{x x} \dot{p}+\left(I_{z z}-I_{y y}\right) q r-(\dot{r}+p q) I_{x z}+\left(r^{2}-q^{2}\right) I_{y z}+(p r-\dot{q}) I_{x y} \\
+m\left[y_{g}(\dot{w}-u q+v p)-z_{g}(\dot{v}-w p+u r)\right]=K \\
I_{y y} \dot{q}+\left(I_{x x}-I_{z z}\right) r p-(\dot{p}+q r) I_{x y}+\left(p^{2}-r^{2}\right) I_{z x}+(q p-\dot{r}) I_{y z} \\
+m\left[z_{g}(\dot{u}-v r+w p)-x_{g}(\dot{w}-u q+v p)\right]=\mathrm{M} \\
I_{z z} \dot{r}+\left(I_{y y}-I_{x x}\right) p q-(\dot{q}+r p) I_{y z}+\left(q^{2}-p^{2}\right) I_{x y}+(r q-\dot{p}) I_{z x} \\
+m\left[x_{g}(\dot{v}-w p+u r)-y_{g}(\dot{u}-v r+w q)\right]=\mathrm{N}
\end{gathered}
$$

Set

$v=\left[\begin{array}{llllll}u & v & w & p & q & r\end{array}\right]^{T}$ as the generalized velocity vector in body frame. $\tau=\left[\begin{array}{llllll}F_{x} & F_{y} & F_{z} & K & M & N\end{array}\right]^{T}$ as the generalizedforcevector in body frame.

We make the equation(29) be written as the following matrix vector form

$M \dot{v}+C(v) v=\tau$

Among them, $M$ and $C(v)$ are the inertial matrix of rigid body system and the coriolis centripetal matrix respectively.

The inertia matrix expression of the rigid body is 


$$
M=\left[\begin{array}{cccccc}
m & 0 & 0 & 0 & m z_{g} & -m y_{g} \\
0 & m & 0 & -m z_{g} & 0 & m x_{g} \\
0 & 0 & m & m y_{g} & -m x_{g} & 0 \\
0 & -m z_{g} & m y_{g} & I_{x x} & -I_{x y} & -I_{x z} \\
m z_{g} & 0 & -m x_{g} & -I_{y x} & I_{y y} & -I_{y z} \\
-m y_{g} & m x_{g} & 0 & -I_{z x} & -I_{z y} & I_{z z}
\end{array}\right]
$$

The coriolis centripetal matrix expression is

$$
\begin{aligned}
& C(v)=\left[\begin{array}{ll}
C_{11} & C_{12} \\
C_{21} & C_{22}
\end{array}\right] \\
& C_{11}=\left[\begin{array}{lll}
0 & 0 & 0 \\
0 & 0 & 0 \\
0 & 0 & 0
\end{array}\right] \\
& C_{12}=\left[\begin{array}{ccc}
m\left(r z_{g}+q y_{g}\right) & m\left(w-q x_{g}\right) & -m\left(v+r x_{g}\right) \\
-m\left(w+p y_{g}\right) & m\left(r z_{g}+p x_{g}\right) & m\left(u-r y_{g}\right) \\
m\left(v-p z_{g}\right) & -m\left(u+q z_{g}\right) & m\left(q y_{g}+p x_{g}\right)
\end{array}\right] \\
& C_{21}=\left[\begin{array}{ccc}
-m\left(r z_{g}+q y_{g}\right) & m\left(w+p y_{g}\right) & m\left(p z_{g}-v\right) \\
m\left(q x_{g}-w\right) & -m\left(r z_{g}+p x_{g}\right) & m\left(u+q z_{g}\right) \\
m\left(v+r x_{g}\right) & m\left(r y_{g}-u\right) & -m\left(q y_{g}+p x_{g}\right)
\end{array}\right] \\
& C_{22}=\left[\begin{array}{ccc}
\left(p I_{z x}+q I_{z y}-r I_{z z}\right) & \left(p I_{y x}-q I_{y y}+r I_{y z}\right) \\
\left(-p I_{y x}+q I_{y y}-r I_{y z}\right) & \left(-p I_{x x}+q I_{x y}+r I_{x z}\right) & 0
\end{array}\right]
\end{aligned}
$$

\section{Force and moment}

The rigid body dynamics describes the motion of four rotor UAV under the action of force and moment. These knowledges of generalized forces make us solve the rigid body vector equation about the variable $v$. That is to say, these solve the system of six difference equations about the variables $u, v, w, p, q, r$. Once these variables are known, we can use the kinematics to express the linear displacement $p_{o}^{I}$ and the attitude of UAV $\Theta$ which is relative to the inertial coordinate system enough. Then we have a concrete analysis on a variety of forces and moments which act on UAV.

\section{Gravity}

When we consider the influence of gravity, we make the gravity act on the centroid and go along the $z$ axis of inertial frame.

$$
F_{g}=R^{T}\left[\begin{array}{c}
0 \\
0 \\
-m g
\end{array}\right]
$$

Among them, $R^{T}$ is the transformation Matrix between inertial frame and body frame. Because the point of action is in the center of gravity, the gravity doesn't produce any moment.

2. Thrust 
The thrust $f$ acts on the center of the $f_{c}$ th electric motor, and the distance from it to the centroid is $\Lambda$. In addition, the direction goes upward along the axis: $f_{j} \geq 0, j=1,2,3,4$. The thrust can be considered as the input of four rotor system, because it can change the rotating speed of rotor. Seen from the physical perspective, the speed of each motor is limited, because the value of each thrust also has a upper bound: $f_{j} \leq f_{\text {MAX }}, j=1,2,3,4$. The imbalance of the force $f_{j}, j=1,3$ or $j=2,4$ leads to go along the moment in the vertical direction included the force $f_{j}$ plane. This moment makes the four rotor rotate along the axis $x_{B}$ and the axis $y_{B}$. About the rotation of the axis $z_{B}$ is the result caused by the unbalance action of clockwise and counterclockwise pulling force moment.

The forces marked as $f_{1}, f_{2}, f_{3}, f_{4}$ are the upward lift force caused by the propeller. Because they are the forces which are controlled to input in the body frame, they can only have the nonzero elements in the direction of axis $Z_{B}$.

$$
f_{j}=\left[\begin{array}{l}
0 \\
0 \\
f_{j}
\end{array}\right]
$$

The thrust expression can be expressed in the following form:

$$
f_{j}=C_{T} \rho A r^{2} \omega_{j}^{2}
$$

Among them, $C_{T}$ is the thrust coefficient, $\rho$ is the air density, $A$ is the rotor disc area, $r$ is the rotor radius, $\omega_{j}$ is the rotational speed of electric motor $j$.

Mark

$$
d_{j}=\left[\begin{array}{l}
d_{j, x} \\
d_{j, y} \\
d_{j, z}
\end{array}\right]
$$

Each element represents the deviation which the center of the electric motor $j$ relative to the center of gravity. Because the moment expression of force $f_{j}$ about the body frame is

$$
m_{j}=f_{j} \times d_{j}
$$

3. Propeller Resistance Moment

The aerodynamic force which act on each electric motor blade and the direction of thrust (The blade responds to the rotation.) is orthogonal, and its production and addition are included in the model. This kind of moment is called that the direction of resistance moment goes along the axis $Z_{B}$.

$$
q_{j}=\left[\begin{array}{c}
0 \\
0 \\
q_{j}
\end{array}\right]
$$

According to the deduction method of thrust, we can derive the resistance moment expression similarly.

$$
q_{j}=C_{Q} \rho A r^{3} \omega_{j}\left|\omega_{j}\right|
$$

Among them $C_{Q}$ is the resistance coefficient.

\section{Air Resistance}

The reasonfor air friction is that the direction of force relative to air speed acts on four rotator UAV. And it represents the translational motion and rotational motion if the air acts on the air vehicle in the heavy air. Translational resistance and rotational resistance are usually very small and follow the stokes' law approximately. The translational air resistance $k_{t} v_{o}^{b}$ and the rotating air 
resistance moment $k_{r} \omega_{n b}^{b}$ are assumed to be linearly proportional to the velocity $v_{o}^{b}$ and the instantaneous angular velocity $\omega_{n b}^{b}$ respectively.

Model Equation Sets

We make use of the above rigid body kinematics equations, rigid body dynamics, force and moment which act on the four rotor UAV to be able to represent the UAV model. The vector form of the full model is shown as follows.

$$
\begin{aligned}
& v_{o}^{I}=R v_{o}^{B} \\
& \dot{\Theta}=T \omega_{n b}^{b} \\
& M \dot{v}+C(v) v=\left[\begin{array}{c}
F_{o}^{B} \\
\tau_{0}^{B}
\end{array}\right]=\left[\begin{array}{c}
\sum_{j=1}^{4} f_{b, j}+F_{g}-k_{t} v_{0}^{B} \\
\sum_{j=1}^{4}\left(q_{j}+m_{j}\right)-k_{r} \omega^{B}
\end{array}\right]
\end{aligned}
$$

Among them, $F_{g}$ represents the gravity, $q_{j}$ represents the rotor resistance, $m_{j}$ represents the thrust moment.

The complete expressions mentioned above apply to the four rotor dynamic description on most flight conditions, and then under some assumption conditionswe can make them be simplified so as to get the simpler equations for the purpose of simulation and control.

Usually the four rotor UAV is built relatively to the body frame mentioned above, and the center of gravity is close to the center of geometry. The distance from the electric motor to the center of gravity is $l$. According to this kind of design, the inertia matrix can be simplified as

$$
I=\left[\begin{array}{ccc}
I_{x x} & 0 & 0 \\
0 & I_{y y} & 0 \\
0 & 0 & I_{z z}
\end{array}\right]
$$

And

we

can think that , $d_{1}=-d_{3}=\left[\begin{array}{lll}l & 0 & 0\end{array}\right]^{T} \quad d_{1}=-d_{3}=\left[\begin{array}{lll}l & 0 & 0\end{array}\right]^{T},-d_{2}=d_{4}=\left[\begin{array}{lll}0 & l & 0\end{array}\right]^{T}-d_{2}=d_{4}=\left[\begin{array}{lll}0 & l & 0\end{array}\right]^{T}$.

The directions of thrusts all go along the direction of axis $z_{B}$, then the overall lift force can be represented as

$$
\sum_{j=1}^{4} f_{b, j}=\left[\begin{array}{c}
0 \\
0 \\
f_{1}+f_{2}+f_{3}+f_{4}
\end{array}\right]
$$

Because the actuating force which acts on the four rotor, the moment can be expressed as

$$
\sum_{j=1}^{4}\left(q_{j}+m_{j}\right)=\left[\begin{array}{c}
l\left(f_{4}-f_{2}\right) \\
l\left(f_{3}-f_{1}\right) \\
d\left(f_{1}-f_{2}+f_{3}-f_{4}\right)
\end{array}\right]
$$

Among them $l$ is the arm length, $d$ is the coefficient ratio of blade resistance to thrust.

We use the symbol

$$
u_{f}=f_{1}+f_{2}+f_{3}+f_{4}
$$

and

$$
\left[\begin{array}{c}
\tau_{p} \\
\tau_{q} \\
\tau_{r}
\end{array}\right]=\left[\begin{array}{c}
l\left(f_{4}-f_{2}\right) \\
l\left(f_{3}-f_{1}\right) \\
d\left(f_{1}-f_{2}+f_{3}-f_{4}\right)
\end{array}\right]
$$

The simplified form of system is

$$
\ddot{y}=\frac{1}{m}\left[\left(C_{\phi} S_{\theta} S_{\psi}-C_{\psi} S_{\phi}\right) u_{f}-k_{t} \dot{y}\right] \ddot{y}=\frac{1}{m}\left[\left(C_{\phi} S_{\theta} S_{\psi}-C_{\psi} S_{\phi}\right) u_{f}-k_{t} \dot{y}\right]
$$




$$
\begin{aligned}
& \ddot{z}=\frac{1}{m}\left[\left(C_{\theta} C_{\phi}\right) u_{f}-m g-k_{t} \dot{z}\right] \ddot{z}=\frac{1}{m}\left[\left(C_{\theta} C_{\phi}\right) u_{f}-m g-k_{t} \dot{z}\right] \\
& \dot{p}=\frac{1}{I_{x x}}\left[-k_{r} p-q r\left(I_{z z}-I_{y y}\right)+\tau_{p}\right] \dot{p}=\frac{1}{I_{x x}}\left[-k_{r} p-q r\left(I_{z z}-I_{y y}\right)+\tau_{p}\right] \\
& \dot{r}=\frac{1}{I_{z z}}\left[-k_{r} r-p q\left(I_{y y}-I_{x x}\right)+\tau_{r}\right] \dot{r}=\frac{1}{I_{z z}}\left[-k_{r} r-p q\left(I_{y y}-I_{x x}\right)+\tau_{r}\right] \\
& \dot{\phi}=p+q S_{\phi} T_{\theta}+r C_{\phi} T_{\theta} \dot{\phi}=p+q S_{\phi} T_{\theta}+r C_{\phi} T_{\theta} \\
& \dot{\theta}=q C_{\phi}-r S_{\phi} \dot{\theta}=q C_{\phi}-r S_{\phi} \\
& \dot{\psi}=\frac{1}{C_{\theta}}\left[q S_{\phi}+r C_{\phi}\right] \dot{\psi}=\frac{1}{C_{\theta}}\left[q S_{\phi}+r C_{\phi}\right] \\
& \text { Among them } \quad S_{(\cdot)}, C_{(\cdot)} \quad \text { and }
\end{aligned}
$$$$
u_{1}=-\left(\left(x_{9}-z_{d}\right)+f_{z}(x)+c_{z 1}\left(x_{12}-\dot{z}_{d}\right)+c_{z 2} e_{z 2}-\ddot{z}_{d}\right) /\left(b_{z}(x) \hat{\theta}_{1}\right) \quad \text { and }
$$

$\dot{\hat{\theta}}_{1}=\rho\left(x_{12}+c_{z 1}\left(x_{9}-z_{d}\right)-\dot{z}_{d}\right) b_{z}(x) u_{1}$ correspondingly. The equation set obtained represents a new UAV system, and the inputs of system models are $\dot{V}_{z 3}=-c_{z 1} e_{z 1}^{2}-c_{z 2} e_{z 2}^{2} \leq 0$ respectively. However, in the actual situation the inputs of system models are four thrusts, namely $z$.

$$
\left[\begin{array}{l}
u_{f} \\
\tau_{p} \\
\tau_{q} \\
\tau_{r}
\end{array}\right]=\left[\begin{array}{cccc}
1 & 1 & 1 & 1 \\
0 & -l & 0 & l \\
-l & 0 & l & 0 \\
d & -d & d & -d
\end{array}\right]\left[\begin{array}{l}
f_{1} \\
f_{2} \\
f_{3} \\
f_{4}
\end{array}\right]
$$

According to the equation mentioned above, we can make the four control input components be represented with the vector as $u=\left[\begin{array}{llll}u_{1} & u_{2} & u_{3} & u_{4}\end{array}\right]^{T}$. Thus, when the four rotor UAV is trouble-free, the relationship between controlled quantity $u$ and the thrust $f$ is

$$
\begin{aligned}
& u=T f \\
& {\left[\begin{array}{l}
u_{1} \\
u_{2} \\
u_{3} \\
u_{4}
\end{array}\right]=\left[\begin{array}{cccc}
1 & 1 & 1 & 1 \\
0 & -l & 0 & l \\
-l & 0 & l & 0 \\
d & -d & d & -d
\end{array}\right]\left[\begin{array}{l}
f_{1} \\
f_{2} \\
f_{3} \\
f_{4}
\end{array}\right]}
\end{aligned}
$$

Among them, $l=0.255, d=0.0024$.

When we consider the actuator of four rotor UAV has the multiplicative fault, its model is $f=\Lambda_{p} f_{c}$

Among them, $f$ represents the control instruction, $f_{c}$ represents the actual output thrust of actuator. $\Lambda_{p}$ is the effectiveness matrix of actuator, and the concrete form is

$$
\Lambda_{p}=\left[\begin{array}{cccc}
\lambda_{1} & 0 & 0 & 0 \\
0 & \lambda_{2} & 0 & 0 \\
0 & 0 & \lambda_{3} & 0 \\
0 & 0 & 0 & \lambda_{4}
\end{array}\right]
$$

The model mentioned above can be used to describe the dynamic state of most of the four rotor UAV. Related parameters are given in the form below. 
Table 1 The Related Parameters of four rotor UAV

\begin{tabular}{lll}
\hline Parameters & Value & Unit \\
\hline$l$ & 0.255 & $\mathrm{~m}$ \\
$m$ & 0.5 & $\mathrm{~kg}$ \\
$I_{x x}$ & $5.9 * 10-3$ & $\mathrm{~kg} \cdot \mathrm{m}^{2}$ \\
$I_{y y}$ & $5.9 * 10-3$ & $\mathrm{~kg} \cdot \mathrm{m}^{2}$ \\
$I_{z z}$ & $1.16 * 10-3$ & $\mathrm{~kg} \cdot \mathrm{m}^{2}$ \\
$d$ & $2.4 * 10-3$ & $\mathrm{l}$ \\
$g$ & 9.81 & $\mathrm{~m} \cdot \mathrm{s}^{2}$ \\
\hline
\end{tabular}

\section{Conclusion}

We first define the coordinate system that is commonly used in the four rotor UAV, and in this way we get the transformational relation of each vector for the four rotor UAV in the body frame and the inertial frame in the paper. Then for the purpose of having an easy modeling, we adopt the newton - euler method to get the rigid body dynamic equations of four rotor model in the paper. We then further analyze all kinds of forces and moments which act on the four rotor UAV in details again in the paper. Finally we deduce the complete expression of overall mathematical model for four rotor UAV and make it be simplified in the paper. In addition, we also put forward the model after the actuator of four rotor UAV has the multiplicative fault.

\section{References}

[1] Nie Bowen. Research on the Modeling and the Control Method of Microminiature Four Rotor UAV Helicopter [D]. Changsha: National University of Defense Technology, 2006.

[2] Tarek Madani A B. Backstepping Control for a Quadrotor Helicopter [C]. In IEEE/RSJ International Conference on Intelligent Robots and Systems. 2006: 3255-3260.

[3] AAl-Hiddabi S. Quadrotor Control Using Feedback Linearization with Dynamic Extension [C]. In Proceeding ofthe 6th International Symposium on Mechatron-ics and its Applications (ISMA09). UAE, 2009: 82-84.

[4] Bouabdallah S, Noth A, Siegwan R. PID vs LQ Control Techniques Applied to an Weight augmentation High energy conruniption Indoor Micro Quadrotor[C]. In Proceedings of 2004 1EEEIRS.J Internationel Conference On Intelligent Robots and Systems. Japan, 2004: 2451-2456. 